\title{
Chemoselectivity Switching through Coupled Equilibria
}

\author{
Thamon Puangsamlee and Ognjen Š. Miljanić* \\ Department of Chemistry, University of Houston, Houston, Texas 77204-5003, United States
}

\begin{abstract}
Complex mixtures are found in biological and petrochemical feedstocks, and in the primordial soup implicated in the origins of life. Reacting individual compounds within these mixtures is challenging because of the difficulty in controlling the chemoselectivity of such reactions. We show that the selectivity of imine oxidation can be controlled within doubly dynamic combinatorial libraries, wherein two coupled equilibria - those of imine exchange and the diaza-Cope rearrangement-determine whether the most oxidizable aldehyde precursor is made available to the oxidant or sequestered away from it. Under the slow oxidation conditions, the most electron-rich precursor can traverse the shallow energy landscape and its oxidation product dominates the final mixture. Faster oxidation captures the imine mixture composition, favoring the products derived from electron poorer push-pull imines. Intermediate oxidation rates allow the expression of yet the third possible reaction product. These findings suggest that careful tuning of reactant concentrations and the associated reaction rates can engage individual mixture components into selective reactions even in the absence of specific catalysts.
\end{abstract}

Control of chemoselectivity is among the central problems of synthetic chemistry. ${ }^{1}$ Its ramifications range from solving the mysteries of prebiotic chemistry ${ }^{2}$ to improving the efficiencies of separations and functionalizations of feedstocks derived from complex petroleum and biomass sources. For a given set of reactants, reaction outcome is controlled by thermodynamic and kinetic factors. Often, they work hand-in-hand: the most stable reaction products also form the fastest. Sometimes, they do not, and such reactions can express either their kinetic (fastest formed) or thermodynamic (most stable) products. Are these two extremes the only options? In this Communication, we show that they are not: in a complex mixture of equilibrating precursors, final reaction product can be switched at well by changing the rate of reagent addition. The key to this behavior is the use of coupled equilibria, in which the products of one reversible reaction act as the substrates for another. Coupled equilibria phenomena ${ }^{3}$ have vast relevance to pollution, climate change, ${ }^{4}$ ocean acidification, ${ }^{5}$ and metabolic pathways. ${ }^{6}$ Complex coupled equilibria often give rise to systems-level and emergent phenomena. ${ }^{7}$

Our studies of irreversible reactions operating on imine dynamic combinatorial libraries (DCLs) ${ }^{8}$ have shown that such "messy" precursor mixtures can still be chemoselectively functionalized. As one DCL component reacts the fastest in an irreversible reaction, other library members equilibrate to produce more of it. The net result is the high-yielding functionalization of the most reactive DCL member, which is amplified at the expense of the less reactive members. ${ }^{9}$ Applied iteratively, such amplification leads to kinetic self-sorting ${ }^{10}$ of the DCL into just a handful of fast-reacting components. Self-sorting hinges on the applicability of the Curtin-Hammett principle, ${ }^{11}$ which implies much faster rate of the DCL equilibration compared to the irreversible removal reaction. In such a scenario, relative stabilities of different imines have no bearing on the final reaction products: it is only the relative rates of that irreversible reaction which determine the chemoselectivity. In this study, we manipulated these relative reaction rates, causing a breakdown of the Curtin-Hammett selectivity but in turn revealing a rich reactivity landscape wherein products can be selected by adjusting the rate of the irreversible removal reaction.

Imines formed from 1,2-phenylenediamine (3, Scheme 1) and aromatic aldehydes can be oxidized into corresponding benzimidazoles using relatively elemental iodine $\left(\mathrm{I}_{2}\right)$. This oxidation is faster for imines formed from electron-rich aldehyde and amine components than for those derived from electron-poor precursors. During our studies of oxidative self-sorting of imines, ${ }^{12}$ we noticed that the product distribution could additionally be influenced by the rate of oxidant addition. The reaction of equimolar amounts of 4-nitrobenzaldehyde (1), 4-methoxybenzaldehyde (2), and 3 generated a mixture of imines $\mathbf{4}$ and $\mathbf{5}$, along with some leftover aldehydes. When this mixture was treated with $\mathrm{I}_{2}$ as the oxidant, the imines were converted into benzimidazoles 6 and 7. The ratio of the two products depended on the rate of $I_{2}$ addition. If $I_{2}$ was added very slowly-over $120 \mathrm{~h}$ - the methoxy-substituted 6 constituted $87 \%$ of the product mixture. On the other hand, instantaneous $(<2 \mathrm{~min}) \mathrm{I}_{2}$ addition resulted in benzimidazole 7 as the dominant product (76\%). This selectivity switching could be explained as follows. The intermediate imine mixture has more of the nitro-substituted imine 4: its pushpull electronic nature means it is both more stable and more quickly formed than the methoxy-substituted $\mathbf{5}$. If the oxidation is quick, it captures this composition of imine precursors in the final ratio of benzimidazole products. However, if the oxidation is very slow, the

Scheme 1. Oxidation of the mixture of imines 4 and 5 produces 6 and 7 in a ratio dependent on the rate of the $I_{2}$ addition.

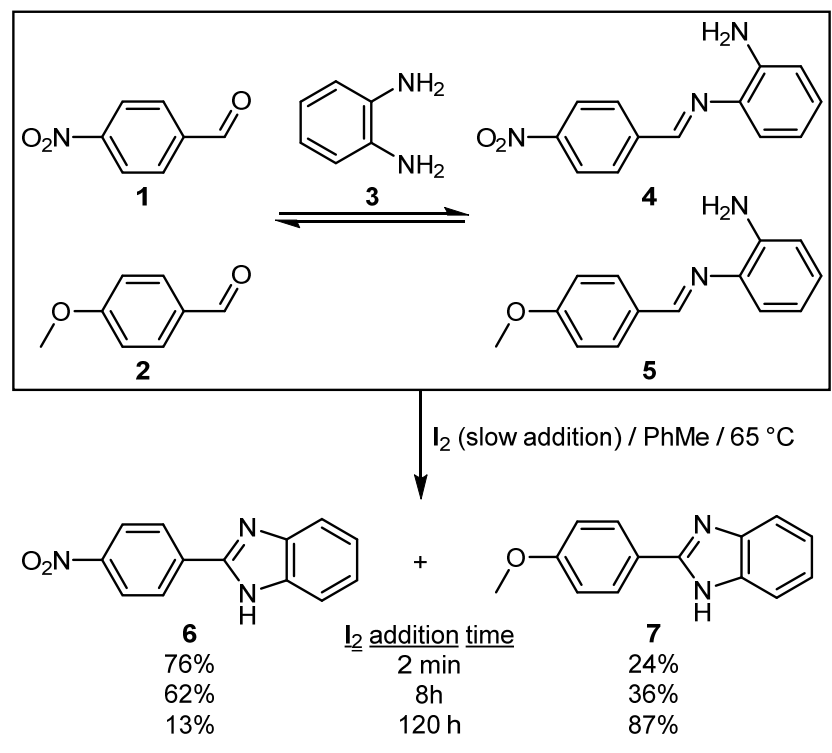


dynamic imine mixture has enough time to continuously replenish the electron-rich imine $\mathbf{5}$ which is being preferentially consumed by its faster oxidation. Slower to oxidize, 4 instead releases its 1,2-phenylenediamine component to create more of $\mathbf{5}$, and the product distribution switches.

Intrigued by this result, we set out to determine whether such selectivity switching could be further controlled. To do so, we designed a dynamic system which operates using two reversible reactions: imine exchange and the diaza-Cope rearrangement of diimines of the general structural type $\mathbf{8}$ (Scheme 2 ). Work by Vögtle ${ }^{13}$ and others ${ }^{14}$ has shown that the diaza-Cope equilibrium favors imines derived from salicylaldehyde (such as $\mathbf{8}$ ) over their rearranged isomers (such as 9), presumably on account of their greater stabilization by $[\mathrm{N} \cdots \mathrm{H}-\mathrm{O}]$ hydrogen bonding. This equilibrium position means that the salicylaldehyde component (highlighted in red in Scheme 2) can be directly exchanged from its imine $\mathbf{8}$ by a reaction with 3 to produce oxidizable imine 10. The more electron-rich 2,4-dimethoxybenzaldehyde (highlighted in blue) cannot directly engage into an oxidation. Instead, 8 must first undergo diaza-Cope rearrangement into the less favored isomer 9 and only then can 9 exchange with 3 to produce the oxidizable imine 11. Being thus onceremoved from the immediate exchange, the electron-rich 2,4-dimethoxybenzaldehyde component must traverse a longer reaction pathway to engage into an oxidation and may not have enough time to do so if oxidation is too fast. Indeed, $\mathrm{I}_{2}$ addition in 1 min generated effectively only the benzimidazole $\mathbf{1 2}$ derived from salicylaldehyde (98:2 selectivity over 13). As the $I_{2}$ addition was slowed down, 2,4dimethoxybenzaldehyde had enough time to travel across the

Scheme 2. Oxidation of a mixture of 3,8 , and 9 generates 12 and 13 in a ratio which is dependent on the rate of $I_{2}$ addition.

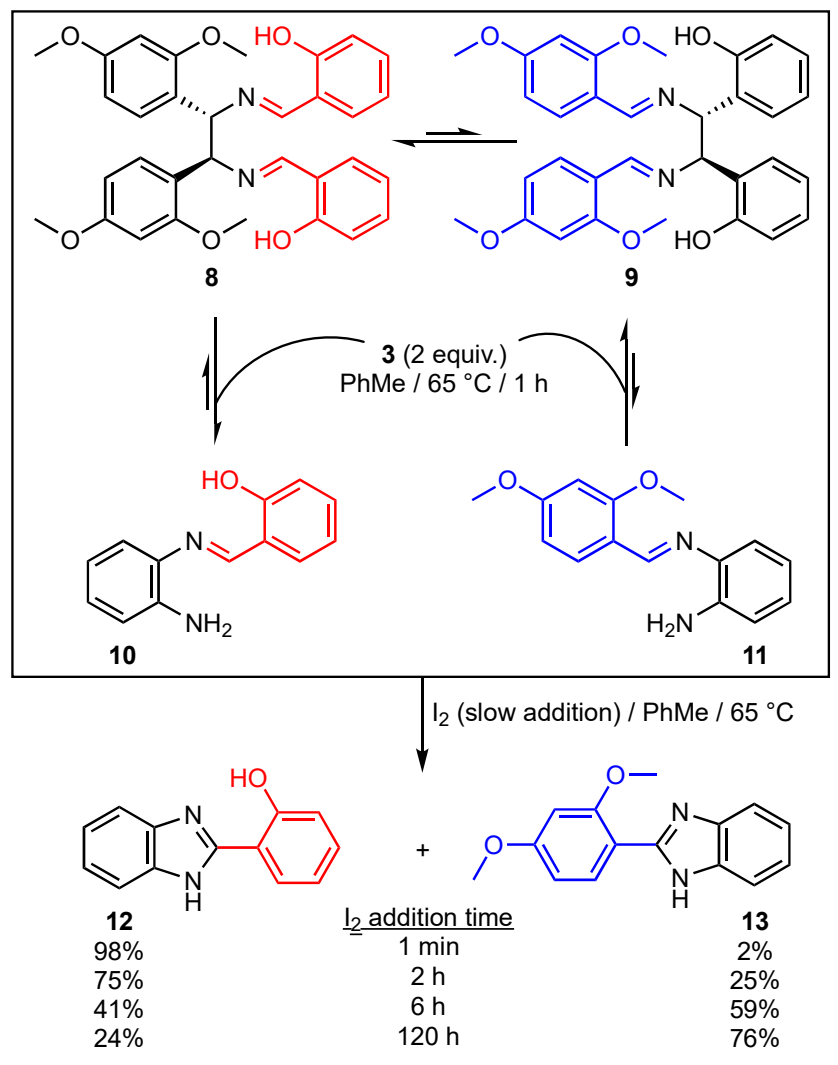

shallow energy landscape and the mole fraction of its oxidation product (13) in the final product mixture increased to $25 \%, 59 \%$, and finally $76 \%$ if $\mathrm{I}_{2}$ was added over $2 \mathrm{~h}, 6 \mathrm{~h}$, or $120 \mathrm{~h}$, respectively.

To establish whether this selectivity switching is general, we examined several other diimines analogous to 8 and $\mathbf{9}$, which related to each other through diaza-Cope rearrangement (Scheme 3). Salicylaldimines $14 a-e$ were preferred over their rearranged isomers 15a-e regardless of the substituents $R$, and a previous study has shown that the diaza-Cope equilibration slows down for electrondonating R groups. ${ }^{14 a}$ The addition of 3 once again initiated the increase in the complexity of this dynamic mixture, as imines $\mathbf{1 0}$ and 16a-d/4 could now be formed, along with partially exchanged species not shown in Scheme 3. Iodine addition then begun, either very slowly or instantaneously. As in the previous experiment, the outcomes of these reactions were dependent on the rate of $\mathrm{I}_{2}$ addition, but this dependence varied based on the substitution in the starting imines 14/15. In the parent system 14a/15a $(R=H)$, slower addition mildly increased the ratio of product $17 \mathbf{a}$ to product 12 , switching from a relative ratio of 1:4.47 to 1:3.29. Selectivity switching observed in Scheme 2 was replicated with the $\mathbf{1 4 b} / \mathbf{1 5 b}(\mathrm{R}=\mathrm{Me})$ couple; the benzimidazole obtained from the electron-rich $\mathbf{1 6} \mathbf{b}$ dominated the product mixture under slow addition conditions, while $\mathbf{1 2}$

Scheme 3. Selectivity switching in imine oxidation as a function of substitution and $I_{2}$ addition rate.

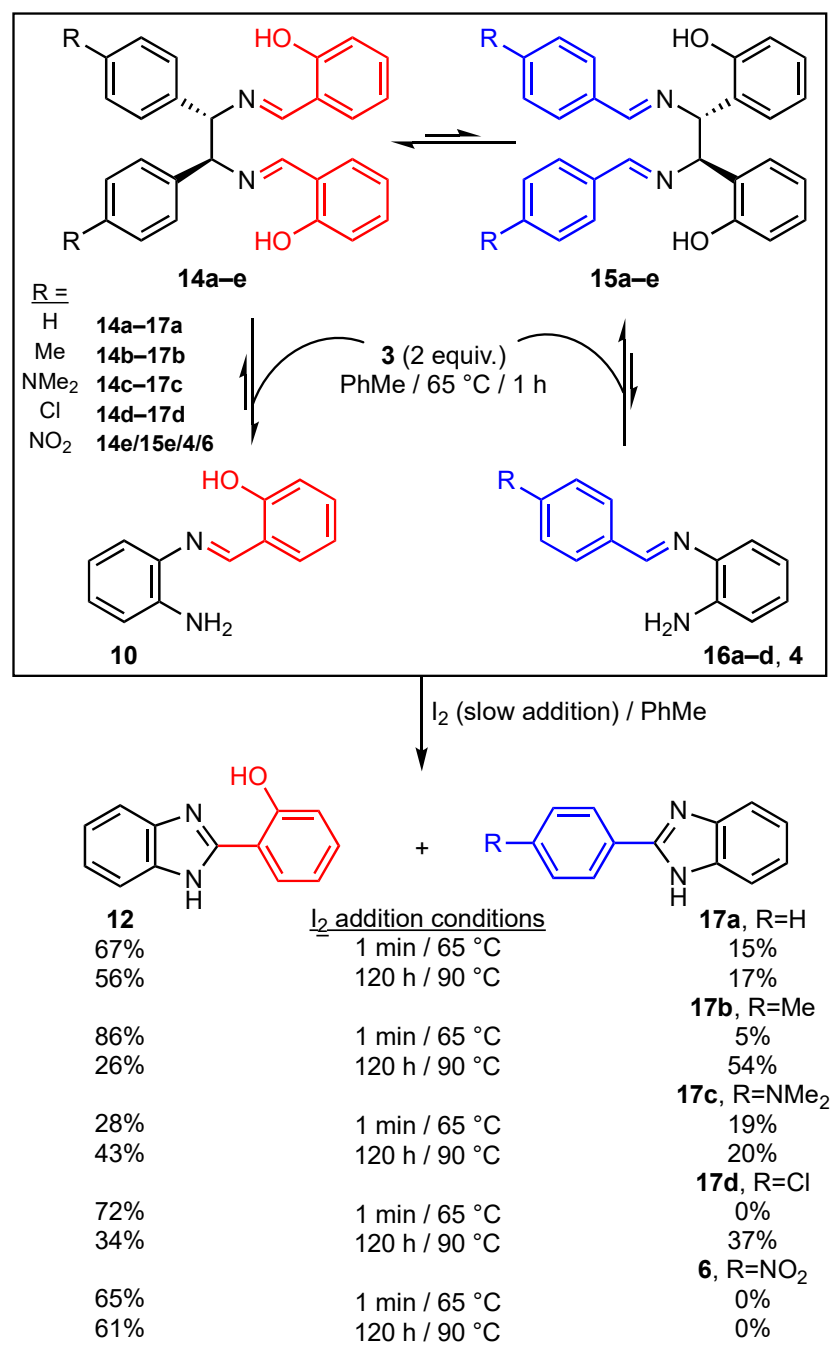


was the main product if $\mathrm{I}_{2}$ was added instantaneously. In compounds 14c/15c, the result was puzzling: the significantly more electronrich para- $N, N$-dimethylamino substituent failed to yield $\mathbf{1 6} \mathbf{c}$ as the dominant product even at long addition times. Possible explanation for this aberration can be found in the slowdown of the diaza-Cope rearrangement in systems with electron-rich substituents. ${ }^{14 a}$ This slowing may have effectively shut down the pre-equilibration, making the imine library not dynamic anymore-and the product ratio independent of the $I_{2}$ addition rate. The example of $14 d / 15 d$ $(\mathrm{R}=\mathrm{Cl})$ offered another interesting conclusion. As expected, 12 dominated under fast addition conditions, but the switch to $\mathbf{1 7} \mathbf{d}$ as the major product with slower $I_{2}$ addition was surprising at first glance. Tentatively, this behavior can be rationalized as follows. The chlorine substituent in the para-position is weakly electron-withdrawing, with a Hammett parameter of +0.23 . Ortho-substitutents are generally not used in Hammett correlations because of steric effects, but an $\mathrm{OH}$ group in a meta position has a Hammett value close to that of para-Cl: $+0.12 .{ }^{15}$ Stated differently, the $\mathrm{OH}$ group influences the electronics of precursor imines by its electron-donating resonance effect, but also as an electron-withdrawing inductive acceptor. Finally, the electron-withdrawing $\mathrm{NO}_{2}$ group in 14e/15e led to identical product mixtures regardless of the $\mathrm{I}_{2}$ addition rate: 12 was the only product, with no $\mathbf{6}$ observed in either case. Nitro-substituted 4 oxidizes so much slower than 10, that even allowing extra time for pre-equilibration did not make a difference in the amount of produced 6.

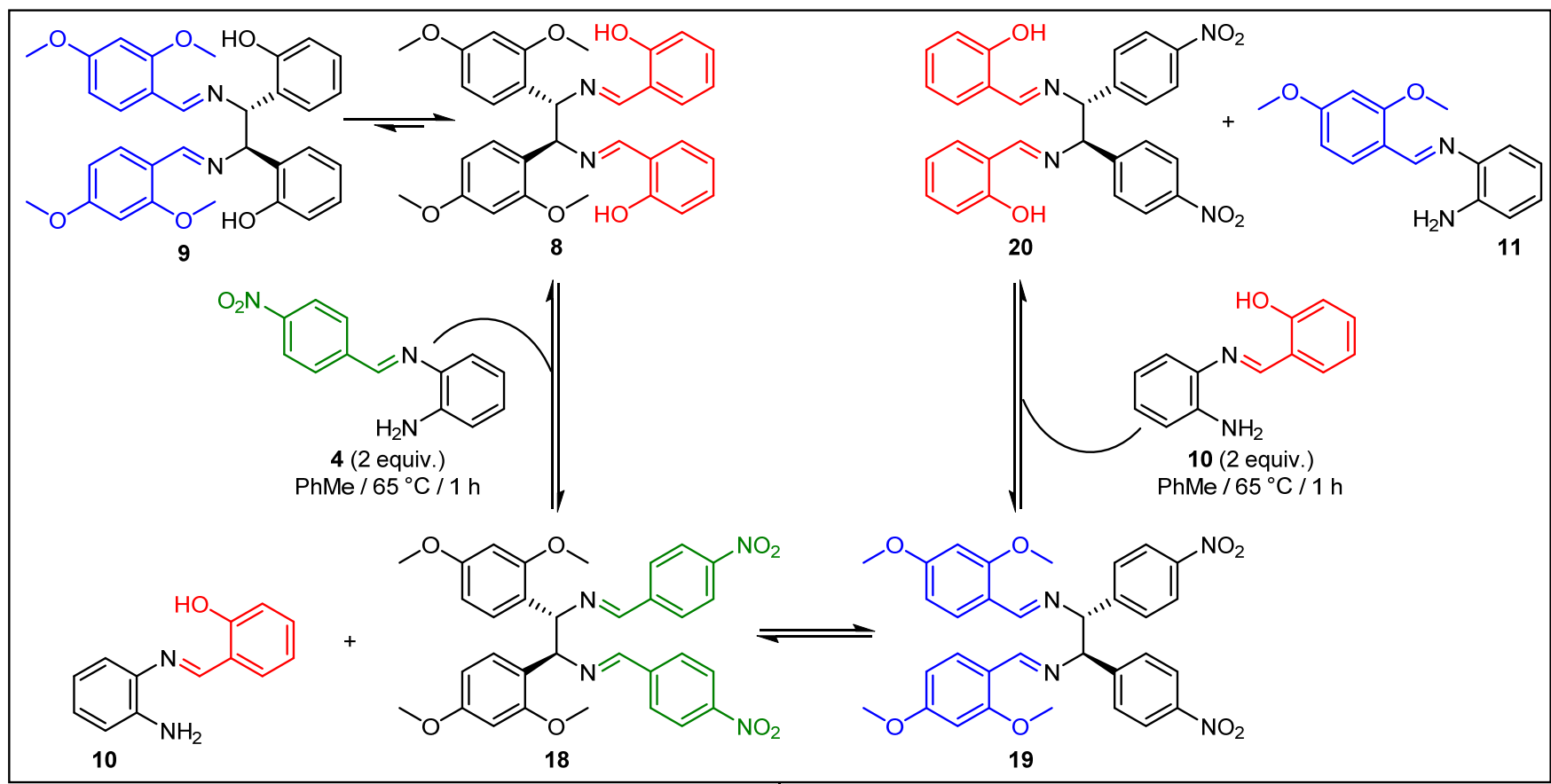

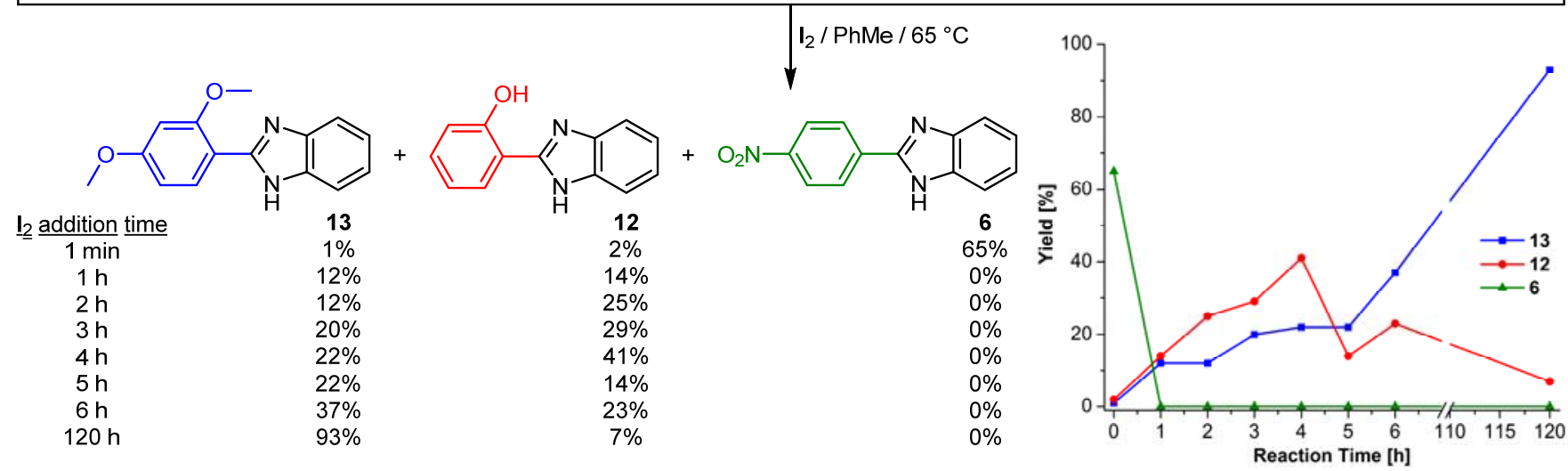

Scheme 4. Three-way switching of chemoselectivity in imine oxidation as a function of $I_{2}$ addition rate.

Encouraged by the confirmation of this hypothesis, we speculated that a three-way selectivity switching may be possible as well. The experiments aimed at testing this hypothesis exposed the diazaCope precursor 8 not to 1,2-phenylenediamine (3), but instead to its imine with 4-nitrobenzaldehyde (4). Three oxidation products could be anticipated from such a DCL (Scheme 4). With very fast oxidant addition, imine 4 would have no time to exchange with 8 and would simply oxidize into 6 . If oxidation was slower, the exchange between 8 and 4 was expected to generate 10 and 18 could then be oxidized into 12 faster than the electron-poorer 4. Finally, if oxidant addition was slower still, diaza-Cope rearrangement of $\mathbf{8}$ or $\mathbf{1 8}$ into 9 or 19 could have occurred. The electron-richest imine 11 could be formed from either of these two latter imines by imine metathesis and its fast oxidation would have produced the third possible product: benzimidazole 13. A series of experiments confirmed this analysis. Instantaneous addition of $\mathrm{I}_{2}$ to the reaction mixture produced only benzimidazole $\mathbf{6}$, derived from the electron-poorest oxidation precursor 4 . As the oxidation was slowed to $2-4 \mathrm{~h}$, salicylaldehydederived 12 dominated the product mixture, with 13 being the minor 
product; under these conditions, no 6 was observed. Oxidant addition over 5-120 h led to the generation of $\mathbf{1 3}$ as the major product, with 12 eventually forming just $7 \%$ of the product mixture.

In conclusion, we have shown that the tuning of relative rates of irreversible and reversible transformations in a DCL can express a variety of its members, ranging from electron-rich to electron-poor. This product selection was accomplished in the absence of any enzyme or synthetic catalysts that could impart selectivity, and the only parameter changed was simply the rate of oxidant addition. These results may offer insights into processes in prebiotic chemistry: multiple components of the "primordial soup" could have selectively reacted under differing spatiotemporal conditions. We have also shown that the diaza-Cope rearrangement can be used as a well-behaved dynamic reaction, thus expanding the arsenal of dynamic combinatorial chemistry. ${ }^{16}$ These results suggest that in sufficiently complex mixtures there may in fact exist a continuum of products ranging from absolute thermodynamic minima to a number of local minima, that can all be addressed by sufficiently fine-tuning the rates of reagent addition as well as other simple physical reaction parameters: concentration, pressure, and temperature. We believe that the use of coupled equilibria can allow virtual dialing-in of reactivity in highly complex libraries.

\section{ASSOCIATED CONTENT}

Supporting Information. Experimental procedures and copies of ${ }^{1} \mathrm{H}$ NMR spectra. This material is available free of charge via the Internet at http://pubs.acs.org.

\section{AUTHOR INFORMATION}

\section{Corresponding Author}

*miljanic@uh.edu

\section{Author Contributions}

T. P. performed all experiments, and analyzed the results together with O. Š. M. Both authors wrote the manuscript and have given approval to the final version of the manuscript.

\section{ACKNOWLEDGMENT}

We acknowledge the support from the Welch Foundation (award E1768) and the University of Houston. O. Š. M. thanks the Alexander von Humboldt Stiftung for supporting his summer stay at the RuprechtKarls-Universität in Heidelberg (Germany), where some of the ideas on which this manuscript is based were conceived.

\section{REFERENCES}

1. Shenvi, R. A.; O'Malley, D. P.; Baran, P. S. Chemoselectivity: The Mother of Invention in Total Synthesis. Acc. Chem. Res. 2009, 42, 530-541

2. Ruiz-Mirazo, K.; Briones, C.; de la Escosura, A. Prebiotic Systems Chemistry: New Perspectives for the Origins of Life. Chem. Rev. 2014, 114, 285-366

3. (a) Atkins, P.; De Paula, J. Atkins' Physical Chemistry. W. H. Freeman; 2006, pp. 200-202. (b) Adamson, R.; Parks, P. C. An Experiment in Coupled Equilibria. J. Chem. Educ. 1971, 48, 120-121.

4. (a) Ferreira, D.; Marshall, J.; Ito, T.; McGee, D. Linking Glacial-Interglacial States to Multiple Equilibria of Climate. Geophys. Res. Lett. 2018, 45, 9160-9170. (b) Schwartz, S. E.; White, W. H. Solubility Equilibria of the Nitrogen Oxides and Oxyacids in Dilute Aqueous Solution. Gordon and
Breach Science Publishers, 1981. (c) Molina, M. J.; Rowland, F. S. Stratospheric Sink for Chlorofluoromethanes: Chlorine Atom-Catalysed Destruction of Ozone. Nature 1974, 249, 810-812.

5. (a) Mongin, M.; Baird, M.; Tilbrook, B.; Matear, R. J.; Lenton, A.; Herzfeld, M.; Wild-Allen, K.; Skerratt, J.; Margvelashvili, N.; Robson, B. J.; Duarte, C. M.; Gustafsson, M. S. M.; Ralph, P. J.; Steven, A. D. L. The Exposure of the Great Barrier Reef to Ocean Acidification. Nat. Commun. 2016, 7, doi.org/10.1038/ncomms10732. (b) Hoegh-Guldberg, O.; Mumby, P. J.; Hooten, A. J.; Steneck, R. S.; Greenfield, P.; Gomez, E.; Harvell, C. D.; Sale, P. F.; Edwards, A. J.; Caldeira, K.; Knowlton, N.; Eakin, C. M.; Iglesias-Prieto, R.; Muthiga, N.; Bradbury, R. H.; Dubi, A.; Hatziolos M. E. Coral Reefs Under Rapid Climate Change and Ocean Acidification. Science 2007, 318, 1737-1742. (c) McNeil, B. I.; Matear, R. J. Climate Change Feedbacks on Future Oceanic Acidification. Tellus $B$ 2007, 59, 191-198.

6. www.genome.ad.jp/kegg. Last accessed on May 20, 2020.

7. (a) Miljanić, O. Š. Small-Molecule Systems Chemistry. Chem. 2017, 2, 502-524. (b) Mattia, E.; Otto, S. Supramolecular Systems Chemistry. Nat. Nanotech. 2015, 10, 111-119. (c) Peyralans, J. J. P.; Otto, S. Recent Highlights in Systems Chemistry. Curr. Opin. Chem. Biol. 2009, 13, 705713. (d) Ludlow, R. F.; Otto, S. Systems Chemistry. Chem. Soc. Rev. 2008, 37, 101-108.

8. (a) Reek, J. N. H.; Otto, S. Dynamic Combinatorial Chemistry; WileyVCH: Weinheim, Germany, 2010. (b) Corbett, P. T.; Leclaire, J.; Vial, L.; West, K. R.; Wietor, J.-L.; Sanders, J. K. M.; Otto, S. Dynamic Combinatorial Chemistry. Chem. Rev. 2006, 106, 3652-3711. (c) Rowan, S. J.; Cantrill, S. J.; Cousins, G. R. L.; Sanders, J. K. M.; Stoddart, J. F. Dynamic Covalent Chemistry. Angew. Chem. Int. Ed. 2002, 41, 898-952. (d) Belowich, M. E.; Stoddart, J. F. Dynamic Imine Chemistry. Chem. Soc. Rev. 2012, 41, 2003-2024

9. (a) Aymeab, J.-F.; Lehn, J.-M. Self-sorting of Two Imine-based Metal Complexes: Balancing Kinetics and Thermodynamics in Constitutional Dynamic Networks. Chem. Sci. 2020, 11, 1114-1121. (b) He, M.; Lehn, J.-M. Time-Dependent Switching of Constitutional Dynamic Libraries and Networks from Kinetic to Thermodynamic Distributions. J. Am. Chem. Soc. 2019, 141, 18560-18569. (c) Osypenko, A.; Dhers, S.; Lehn, J.-M. Pattern Generation and Information Transfer through a Liquid/Liquid Interface in 3D Constitutional Dynamic Networks of Imine Ligands in Response to Metal Cation Effectors. J. Am. Chem. Soc. 2019, 141, 12724-12737. (d) Hafezi, N.; Lehn. J.-M. Adaptation of Dynamic Covalent Systems of Imine Constituents to Medium Change by Component Redistribution under Reversible Phase Separation. J. Am. Chem. Soc. 2012, 134, 12861-12868.

10. (a) He, Z.; Jiang, W.; Schalley, C. A. Integrative Self-Sorting: A Versatile Strategy for the Construction of Complex Supramolecular Architecture. Chem. Soc. Rev. 2015, 44, 779-789. (b) Ji, Q.; Lirag, R. C.; Miljanić, O. Š. Kinetically Controlled Phenomena in Dynamic Combinatorial Libraries. Chem. Soc. Rev. 2014, 43, 1873-1884. (c) Safont-Sempere, M. M.; Fernández, G.; Würthner, F. Self-Sorting Phenomena in Complex Supramolecular Systems. Chem. Rev. 2011, 111, 5784-5814. (d) Osowska, K.; Miljanić, O. Š. Kinetic and Thermodynamic Self-Sorting in Synthetic Systems. Synlett 2011, 1643-1648.

11. Seeman, J. I. Effect of Conformational Change on Reactivity in Organic Chemistry. Evaluations, Applications, and Extensions of CurtinHammett Winstein-Holness Kinetics. Chem. Rev. 1983, 83, 83-134.

12. (a) Osowska, K.; Miljanić, O. Š. Oxidative Kinetic Self-Sorting of a Dynamic Imine Library. J. Am. Chem. Soc. 2011, 133, 724-727. See also: (b) Osowska, K.; Miljanić, O. Š. Self-Sorting of Dynamic Imine Libraries during Distillation. Angew. Chem. Int. Ed. 2011, 50, 8345-8349. (c) Ji, Q.; Miljanić, O. Š. Distillative Self-Sorting of Dynamic Ester Libraries. J. Org. Chem. 2013, 78, 12710-12716. (d) Hsu, C.-W.; Miljanić, O. Š. Adsorption-Driven Self-Sorting of Dynamic Imine Libraries. Angew. Chem. Int. Ed. 2015, 54, 2219-2222. (e) Hsu, C.-W.; Miljanić, O. Š. Kinetically Controlled Simplification of a Multiresponsive $[10 \times 10]$ Dynamic Imine Library. Chem. Commun. 2016, 52, 12357-12359. 
13. Vögtle, F.; Goldschmitt, E. Die Diaza-Cope-Umlagerung. Chem. Ber. 1976, 109, 1-40.

$14 . \quad$ (a) Lee, D.-N.; Kim, H.; Mui, L.; Myung, S.-W.; Chin, J.; Kim, H.-J. Electronic Effect on the Kinetics of the Diaza-Cope Rearrangement. J. Org. Chem. 2009, 74, 3330-3334. (b) Kim, H.; Nguyen, Y.; Yen, C. P.H.; Chagal, L.; Lough, A. J.; Kim, B. M.; Chin, J. Stereospecific Synthesis of $C_{2}$ Symmetric Diamines from the Mother Diamine by Resonance-Assisted Hydrogen-Bond Directed Diaza-Cope Rearrangement. J. Am. Chem. Soc. 2008, 130, 12184-12191. (c) Chin, J.; Mancin, F.; Thavarajah, N.; Lee, D.; Lough, A.; Chung, D. S. Controlling Diaza-Cope Rearrangement Reactions with Resonance-Assisted Hydrogen Bonds. J. Am. Chem. Soc. 2003, 125, 15276-15277.
15. Some trends seem to suggest that an $-\mathrm{OH}$ group in an ortho-position has an electron withdrawing, rather than donating effect. For example, the acidity of hydroxy-substituted benzoic acids is decreased relative to the parent benzoic acid $\left(\mathrm{p} K_{\mathrm{a}}=4.20\right)$ when the $-\mathrm{OH}$ group is in the paraposition $\left(\mathrm{p} K_{\mathrm{a}}=4.28\right)$ but increased when the same group is meta $\left(\mathrm{p} K_{\mathrm{a}}=3.84\right)$ and ortho $\left(\mathrm{p} K_{\mathrm{a}}=2.98\right)$ to the $-\mathrm{COOH}$ functionality. See also: McDaniel, D. H.; Brown, H. C. An Extended Table of Hammett Substitutent Constants Based on the Ionization of Substituted Benzoic Acids. J. Org. Chem. 1958, 23, 420-427.

16. Jin, Y.; Yu, C.; Denman, R. Y.; Zhang, W. Recent Advances in Dynamic Covalent Chemistry. Chem. Soc. Rev. 2013, 42, 6634-6654. 


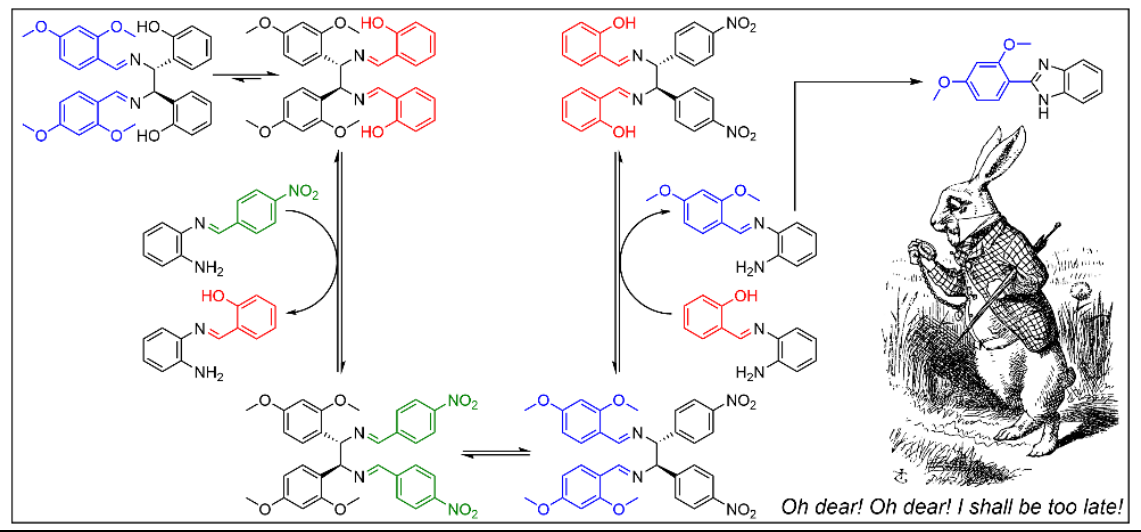

